\title{
ON THE PROPERTIES OF CERTAIN CONTINUED FRACTIONS
}

\author{
EVELYN FRANK
}

1. Introduction. This paper is concerned with a development of the properties of a new class of continued fractions

$$
k_{0} \gamma_{0}+\frac{k_{0}\left(1-\gamma_{0} \bar{\gamma}_{0}\right) z}{\bar{\gamma}_{0} z}-\frac{1}{k_{1} \gamma_{1}}+\frac{k_{1}\left(1-\gamma_{1} \bar{\gamma}_{1}\right) z}{\bar{\gamma}_{1} z}-\frac{1}{k_{2} \gamma_{2}}+\ldots
$$

in which the $k_{p}$ and $\gamma_{p}, p=0,1, \cdots$, are constants and $\left|\gamma_{p}\right| \neq 1$.

A special class of (1.1) are the continued fractions

$$
\gamma_{0}+\frac{\left(1-\gamma_{0} \bar{\gamma}_{0}\right) z}{\bar{\gamma}_{0} z}-\frac{1}{\gamma_{1}}+\frac{\left(1-\gamma_{1} \bar{\gamma}_{1}\right) z}{\bar{\gamma}_{1} z}-\frac{1}{\gamma_{2}}+\ldots,
$$

where $\left|\gamma_{p}\right| \neq 1$. These were first discussed by Schur [6 $]^{1}$ with $\left|\gamma_{p}\right|<1$ in connection with functions bounded in the unit circle.

In $\$ 2$ it is shown that to an arbitrary power series there correspond continued fractions (1.1), and, conversely, to every such continued fraction there corresponds a power series which agrees with the power series for the $2 p$ th and $(2 p+1)$ th approximants of the continued fraction up to and including the terms involving $z^{p}$ and $z^{p-1}$, respectively. An important new feature which is given in $\$ 3$ is the simple algorithm for the expansion of a power series into a continued fraction (1.1), and, conversely, for the computation of the corresponding power series from the continued fraction. Heretofore this computation has been exceedingly cumbersome.

In $\$ 4$ conditions under which these continued fractions converge to the corresponding power series are considered, in particular, if the generating function is meromorphic. In $\$ 5$ convergence regions for the expansions (1.1) are found, and in $\$ 6$ convergence regions for the continued fraction whose approximants are the even and odd approximants of (1.1). In $\$ 7$ certain transformations of (1.1) are discussed.

2. Continued fraction expansions for arbitrary power series. Let the function $f_{0}(z)$ be expanded into a power series

$$
f_{0}(z) \sim c_{00}+c_{01} z+c_{02} z^{2}+\cdots .^{2}
$$

The functions $f_{1}(z), f_{2}(z), \cdots$, are found by the recurrence rela-

Presented to the Society, September 7, 1948; received by the editors January 7, 1952.

${ }^{1}$ Numbers in brackets refer to the bibliography at the end of the paper.

$2 \sim$ denotes a formal expansion. 
tions

$$
f_{p+1}=\frac{\gamma_{p}-f_{p}}{z\left(1-\bar{\gamma}_{p} f_{p}\right)}, \quad f_{p}=\frac{\gamma_{p}-z f_{p+1}}{1-z \bar{\gamma}_{p} f_{p+1}}, \quad \begin{array}{r}
\gamma_{p}=f_{p}(0), \\
p=0,1, \cdots,
\end{array}
$$

and are formally expanded in power series

$$
f_{p}(z) \sim c_{p 0}+c_{p 1} z+c_{p 2} z^{2}+\cdots, \quad p=1,2, \cdots .
$$

The infinite continued fraction expansion (1.2) can then be constructed provided none of the numbers $\left|\gamma_{0}\right|,\left|\gamma_{1}\right|, \cdots$, is equal to unity. The case where $\left|\gamma_{n}\right|=1$ implies that $\left|f_{n}(z)\right| \equiv 1$, and in this case the continued fraction expansion (1.2) is finite. Here it may be shown by a method analogous to that in [2] that $f_{0}(z)$ is a rational function of the form

$$
f_{0}(z)=\frac{\epsilon z^{n} P(z)}{P^{*}(z)}, \quad|\epsilon|=1, \quad P^{*}(z)=\bar{P}\left(z^{-1}\right),
$$

where $P^{*}(z)$ is any polynomial of degree $n$ which does not vanish on the unit circle or is everywhere equal to 1.

The numbers $\gamma_{p}$, which are rational functions of $c_{00}, c_{01}, \cdots$, can be computed from the recurrence formulas for the approximants $A_{p}(z) / B_{p}(z)$ of the continued fraction (1.2) and the determinant formula

$$
A_{2 p+2}(z) B_{2 p}(z)-A_{2 p}(z) B_{2 p+2}(z)=(-1)^{p} \gamma_{p+1} z^{p+1} \prod_{i=0}^{p}\left(1-\gamma_{i} \bar{\gamma}_{i}\right)
$$

by equating coefficients of $z^{p+1}$ in

$$
\begin{array}{r}
f_{0}(z) B_{2 p}(z)-A_{2 p}(z)=-\gamma_{p+1} z^{p+1} \prod_{i=0}^{p}\left(1-\gamma_{i} \bar{\gamma}_{i}\right)+\cdots \\
p=0,1, \cdots .
\end{array}
$$

Thus one finds

$$
\begin{aligned}
& \gamma_{0}=c_{00}, \quad \gamma_{1}=\frac{-c_{01}}{1-c_{00} \bar{c}_{00}}, \\
& \gamma_{2}=\frac{c_{02}\left(1-c_{00} \bar{c}_{00}\right)+\bar{c}_{00} c_{01}^{2}}{\left(1-c_{00} \bar{c}_{00}\right)^{2}-c_{01} \bar{c}_{01}}, \ldots .
\end{aligned}
$$

None of these denominators is zero if none of the numbers $\left|\gamma_{p}\right|$ is equal to 1 . Conversely, the $c_{0 p}$ can be computed as rational functions of the $\gamma_{p}$ : 


$$
\begin{aligned}
& c_{00}=\gamma_{0}, \quad c_{01}=-\gamma_{1}\left(1-\gamma_{0} \bar{\gamma}_{0}\right), \\
& c_{02}=\gamma_{2}\left(1-\gamma_{0} \bar{\gamma}_{0}\right)\left(1-\gamma_{1} \bar{\gamma}_{1}\right)-\gamma_{0} \gamma_{1}^{2}\left(1-\gamma_{0} \bar{\gamma}_{0}\right), \cdots .
\end{aligned}
$$

From (2.6) and the determinant formula

$$
A_{2 p+3}(z) B_{2 p+1}(z)-A_{2 p+1}(z) B_{2 p+3}(z)=(-1)^{p} \bar{\gamma}_{p+1} z^{p+2} \prod_{i=0}^{p}\left(1-\gamma_{i} \bar{\gamma}_{i}\right)
$$

or

$$
\begin{array}{r}
f_{0}(z) B_{2 p+1}(z)-A_{2 p+1}(z)=-z^{p+1} \prod_{i=0}^{p}\left(1-\gamma_{i} \bar{\gamma}_{i}\right)+\cdots, \\
p=0,1, \cdots,
\end{array}
$$

it is seen that the developments in power series of the approximants $A_{2 p}(z) / B_{2 p}(z), p=0,1, \cdots$, and $A_{2 p+1}(z) / B_{2 p+1}(z), p=1,2, \cdots$, coincide with the power series (2.1) up to and including the terms involving $z^{p}$ and $z^{p-1}$, respectively. Furthermore, two finite or infinite continued fractions

$$
\begin{aligned}
& \gamma_{0}+\frac{\left(1-\gamma_{0} \bar{\gamma}_{0}\right) z}{\bar{\gamma}_{0} z}-\frac{1}{\gamma_{1}}+\frac{\left(1-\gamma_{1} \bar{\gamma}_{1}\right) z}{\bar{\gamma}_{1} z}-\frac{1}{\gamma_{2}}+\cdots, \\
& \gamma_{0}^{\prime}+\frac{\left(1-\gamma_{0}^{\prime} \bar{\gamma}_{0}^{\prime}\right) z}{\bar{\gamma}_{0}^{\prime} z}-\frac{1}{\gamma_{1}^{\prime}}+\frac{\left(1-\gamma_{1}^{\prime} \bar{\gamma}_{1}^{\prime}\right) z}{\bar{\gamma}_{1}^{\prime} z}-\frac{1}{\gamma_{2}^{\prime}}+\cdots,
\end{aligned}
$$

have the same corresponding power series (2.1) if and only if $\gamma_{p}=\gamma_{p}^{\prime}$, $p=0,1, \cdots$. Consequently, there is a unique correspondence between the continued fraction (1.2) and the power series (2.1), as stated in the following theorem.

THEOREM 2.1. If numbers $\gamma_{p}$ with moduli different from one are found from the coefficients of a power series (2.1), then there exists a uniquely determined infinite continued fraction of the form (1.2) which corresponds to the power series (2.1). Conversely, to every infinite continued fraction (1.2) in which $\left|\gamma_{p}\right| \neq 1, p=0,1, \cdots$, there corresponds a unique power series (2.1). The power series expansions for $A_{2 p}(z) / B_{2 p}(z), p=0,1, \cdots$, and $A_{2 p+1}(z) / B_{2 p+1}(z), p=1,2, \cdots$, the $2 p$ th and $(2 p+1)$ th approximants of (1.2), agree wrth the power series (2.1) up to and including the terms involving $z^{p}$ and $z^{p-1}$, respectively. The continued fraction (1.2) is finite when $\left|\gamma_{n}\right|=1$, and in this case it represents the rational function (2.4).

If, in the recurrent process for finding the numbers $\gamma_{p}$, one finds that $\left|\gamma_{m}\right|=1$, then $\gamma_{m}$ can be computed for the function $f_{m} / k_{m}$, and 
$f_{m} / k_{m}$ formally expanded in a power series

$$
\frac{f_{m}}{k_{m}} \sim \frac{c_{m 0}}{k_{m}}+\frac{c_{m 1} z}{k_{m}}+\frac{c_{m 2} z^{2}}{k_{m}}+\cdots,\left|k_{m}\right| \neq 1, k_{m} \neq 0 .
$$

One continues the process for the computation of the numbers $\gamma_{p}$, as outlined above, until another value of $\gamma$, namely $\gamma_{q}$, with modulus 1 is reached. Then, as in (2.9), the formal power series for $f_{q} / k_{q}$, $\left|k_{q}\right| \neq 1$, is formed, $\gamma_{q}$ for $f_{q} / k_{q}$ computed, and the usual process for the computation of the numbers $\gamma_{p}$ continued. In this way, one constructs the infinite continued fraction expansion (1.1) where

$$
\begin{aligned}
\gamma_{0} & =\frac{c_{00}}{k_{0}} \\
\gamma_{1} & =\left[\frac{-c_{01}}{k_{1} k_{0}}\right] /\left[1-\frac{c_{00} \bar{c}_{00}}{k_{0}^{2}}\right], \cdots .
\end{aligned}
$$

For this expansion, one obtains the following theorem by a method analogous to that for Theorem 2.1.

THEOREM 2.2. Corresponding to every power series (2.1) there exist infinite continued fractions of the form (1.1), and, conversely, to every infinite continued fraction (1.1) there corresponds a power series (2.1). The power series expansions for $A_{2 p}(z) / B_{2 p}(z), p=0,1, \cdots$, and $A_{2 p+1}(z) / B_{2 p+1}(z), p=1,2, \cdots$, the $2 p$ th and $(2 p+1)$ th approximants of (1.1), agree with the power series (2.1) up to and including the terms involving $z^{p}$ and $z^{p-1}$, respectively.

3. Algorithm for the expansion of a power series into the continued fraction (1.1). Since the actual computation of the numbers $\gamma_{p}$ in the continued fractions (1.1) and (1.2) is somewhat lengthy, it is desirable that one have a simple algorithm for obtaining these numbers. The following theorem may be used to find easily the continued fraction (1.1) corresponding to an arbitrary power series (2.1), and, conversely, to expand a continued fraction of the form (1.1) into its corresponding power series.

Theorem 3.1. Let $f_{0}(z)$ (2.1) denote an arbitrary power series. Determine polynomials $B_{2 p}(z)=\beta_{0}^{(2 p)}+\beta_{1}^{(2 p)} z+\beta_{2}^{(2 p)} z^{2}+\cdots$ and numbers $\gamma_{p}\left(k_{p}\right), p=0,1, \cdots$, such that $\left|\gamma_{p}\right| \neq 1$ by a proper choice of numbers $k_{p}$, by means of the recurrence formulas

$$
\begin{aligned}
& B_{0}(z)=1, \quad B_{1}(z)=\bar{\gamma}_{0} z, \quad B_{2 p}(z)=k_{p} \gamma_{p} B_{2 p-1}(z)-B_{2 p-2}(z), \\
& B_{2 p+1}(z)=\bar{\gamma}_{p} z B_{2 p}(z)+k_{p}\left(1-\gamma_{p} \bar{\gamma}_{p}\right) z B_{2 p-1}(z), p=1,2, \cdots ;
\end{aligned}
$$


$\gamma_{0}=c_{0} / k_{0}$,

$$
\begin{aligned}
& \left(c_{p+1}, c_{p}, c_{p-1}, \cdots\right)\left(\begin{array}{c}
\beta_{0}^{(2 p)} \\
\beta_{1}^{(2 p)} \\
\beta_{2}^{(2 p)} \\
\vdots \\
\cdot
\end{array}\right)=-k_{p+1} \gamma_{p+1} \prod_{i=0}^{p} k_{i}\left(1-\gamma_{i} \bar{\gamma}_{i}\right), \\
& p=0,1, \cdots .
\end{aligned}
$$

Then (1.1) is a continued fraction corresponding to $f_{0}(z)$. The polynomials $B_{p}(z)$ are the denominators of the approximants of (1.1). The numerators of the approximants are $A_{p}(z)=\delta_{0}^{(p)}+\delta_{1}^{(p)} z+\cdots$, where

$$
\begin{aligned}
& \left(\delta_{0}^{(2 p)}, \delta_{1}^{(2 p)}, \delta_{2}^{(2 p)}, \ldots\right) \\
& =\left(\beta_{0}^{(2 p)}, \beta_{1}^{(2 p)}, \beta_{2}^{(2 p)}, \ldots\right) \quad\left(\begin{array}{c}
\left.c_{0}, c_{1}, c_{2}, \cdots, c_{p}, 0, \ldots\right) \\
0, c_{0}, c_{1}, \cdots, c_{p-1}, 0, \ldots \\
0,0, c_{0}, \ldots, c_{p-2}, 0, \ldots \\
. \ldots
\end{array}\right)
\end{aligned}
$$

and

$$
\begin{aligned}
& \left(\delta_{0}^{(2 p+1)}, \delta_{1}^{(2 p+1)}, \delta_{2}^{(2 p+1)}, \cdots\right) \\
& =\left(\beta_{0}^{(2 p+1)}, \beta_{1}^{(2 p+1)}, \beta_{2}^{(2 p+1)}, \cdots\right)\left(\begin{array}{c}
c_{0}, c_{1}, c_{2}, \cdots, c_{p+1}, 0, \cdots \\
0, c_{0}, c_{1}, \cdots, c_{p}, 0, \cdots \\
0,0, c_{0}, \cdots, c_{p-1}, 0, \cdots \\
\cdots \cdots, \ldots
\end{array}\right) \\
& +\left(0,0, \cdots, \prod_{i=0}^{p} k_{i}\left(1-\gamma_{i} \bar{\gamma}_{i}\right), 0,0, \cdots\right), p=0,1, \cdots
\end{aligned}
$$

(where there are $p+1$ zeros preceding the product). The expansion (1.1) is finite when $f_{0}(z)$ is of the form (2.4), and $k_{p}=1, p=0,1, \cdots$.

One proves this theorem by showing by induction that the continued fraction (1.1) determined by (3.2) satisfies the relation

$$
\begin{array}{r}
f_{0}(z) B_{2 p}(z)-A_{2 p}(z)=-k_{p+1} \gamma_{p+1} z^{p+1} \prod_{i=0}^{p} k_{i}\left(1-\gamma_{i} \bar{\gamma}_{i}\right)+\cdots \\
p=0,1, \cdots .
\end{array}
$$


Formulas (3.3) for the coefficients $\delta^{(p)}$ of $A_{p}(z)$ are found if one equates corresponding powers of $z$ in (3.4) and in

$$
\begin{aligned}
f_{0}(z) B_{2 p+1}(z)-A_{2 p+1}(z)=-z^{p+1} \prod_{i=0}^{p} k_{i}\left(1-\gamma_{i} \bar{\gamma}_{i}\right) & +\cdots, \\
p & =0,1, \cdots .
\end{aligned}
$$

The $A_{p}(z)$ are also given by the recurrence formulas

$$
\begin{aligned}
A_{0}(z) & =k_{0} \gamma_{0}, \quad A_{1}(z)=k_{0} z, \\
A_{2 p}(z) & =k_{p} \gamma_{p} A_{2 p-1}(z)-A_{2 p-2}(z), \\
A_{2 p+1}(z) & =\bar{\gamma}_{p} z A_{2 p}(z)+k_{p}\left(1-\gamma_{p} \bar{\gamma}_{p}\right) z A_{2 p-1}(z), \quad p=1,2, \cdots .
\end{aligned}
$$

Conversely, the relations (3.2) serve to determine $f_{0}(z)(2.1)$ when (1.1) is given.

As an illustration of the algorithm, let

$$
f_{0}(z)=1+z+z^{2}+z^{3}+\cdots\left(=\frac{1}{1-z}\right) .
$$

Let $k_{0}=2$. Then $\gamma_{0}=1 / 2, B_{0}(z)=1$, and

$$
(1) \cdot(1)=-k_{0} k_{1}\left(1-\gamma_{0} \bar{\gamma}_{0}\right) \gamma_{1}=-2 \cdot 3 k_{1} \gamma_{1} / 4 \text {. }
$$

Let $k_{1}=2, \gamma_{1}=-1 / 3, B_{2}(z)=-z / 3-1$, and

$$
(1,1)\left(\begin{array}{c}
-1 \\
-1 / 3
\end{array}\right)=-4 \cdot 3 \cdot 8 k_{2} \gamma_{2} / 4 \cdot 9 \text {. }
$$

For $k_{2}=2, \gamma_{2}=1 / 4$. By a continuation of this process with all $k_{p}=2$, then $\gamma_{p}=(-1)^{p} /(p+2), p=0,1, \cdots$, and the continued fraction corresponding to (3.5) is, after some transformations,

$$
\begin{gathered}
\Sigma_{1} n+\frac{\left(2^{2}-1\right) z}{z}+\frac{\Sigma_{1}^{2} n}{1}+\frac{\left(3^{2}-1\right) z}{z}+\frac{\Sigma_{1}^{3} n}{1} \\
+\frac{\left(4^{2}-1\right) z}{z}+\frac{\Sigma_{1}^{4} n}{1}+\ldots .
\end{gathered}
$$

4. Convergence to the corresponding power series. The conditions under which the continued fractions (1.1) and (1.2), corresponding to the power series (2.1), converge to the value of the power series will now be considered.

THEOREM 4.1. If the infinite continued fraction (1.1) or (1.2) con- 
verges uniformly in a closed region $T$ which contains the origin in its interior, the corresponding power series converges to the same function in and on the boundary of every circle $K$ which lies wholly within $T$ and which has its center at the origin.

The proof is omitted since it is analogous to that given by Perron $[5$, p. 342] for Stieltjes-type continued fractions.

The results of Weierstrass $[10 ; 11]$, and Mittag-Leffler $[3 ; 4]$, concerning general infinite product and infinite series developments for meromorphic functions, would lead one to conjecture that it might be possible to find general continued fraction expansions for such functions. To this end, let (2.1) be a power series expansion of a meromorphic function $F_{0}(z)$. The points $r_{1}, r_{2}, \cdots, r_{m}$ are taken as the poles of $F_{0}(z)$ which are in the finite plane, where $1 \leqq\left|r_{1}\right| \leqq\left|r_{2}\right|$ $\leqq \cdots \leqq\left|r_{m}\right|$. It is assumed that the poles on $|z|=1$ are at most simple poles, since $F_{0}(z)$ can always be made to satisfy this condition by a linear transformation. Then the coefficients $c_{0 p}$ (2.1) of the power series expansion of $F_{0}(z)$ are bounded. Consequently, given such a function $F_{0}(z)$, one may expand it into the continued fraction (1.2) provided the numbers $\gamma_{p}$ (2.2) have moduli different from one, and (1.2) converges to $F_{0}(z)$ according to the following theorem.

ThEOREM 4.2. A meromorphic function $F_{0}(z)$ can be uniquely expanded into and represented in the closed domain

$$
C_{0}:|z| \leqq r<1
$$

by the continued fraction (1.2) if the numbers $\gamma_{p}$ (2.2) have moduli different from one and if the coefficients of the power series expansions for the $2 \lambda$ th approximants $(2 \lambda>N)$ of (1.2) are bounded.

Proof. By equating coefficients of $z^{p}$ in the recurrence formulas (2.2), one obtains

$$
\begin{aligned}
c_{0 p} & =\left(1-\gamma_{0} \bar{\gamma}_{0}\right) c_{1, p-1}-\bar{\gamma}_{0} \sum_{\lambda=1}^{p-1} c_{0, \lambda} c_{1, p-1-\lambda} \\
& =\gamma_{p} \prod_{\lambda=0}^{p-1}\left(1-\gamma_{\lambda} \bar{\gamma}_{\lambda}\right)+K
\end{aligned}
$$

where $K$ depends only on $\gamma_{0}, \bar{\gamma}_{0}, \cdots, \gamma_{p-1}, \bar{\gamma}_{p-1}$. If one puts $\gamma_{\lambda}$ equal to a number of absolute value 1 , then $c_{0 p}$ no longer depends on $\gamma_{\lambda+1}, \bar{\gamma}_{\lambda+1}, \cdots, \gamma_{v}$. In this case $c_{0 p}$ is the coefficient of $z^{p}$ in the development of 


$$
\begin{aligned}
\gamma_{0} & +\frac{\left(1-\gamma_{0} \bar{\gamma}_{0}\right) z}{\bar{\gamma}_{0} z}-\frac{1}{\gamma_{1}}+\frac{\left(1-\gamma_{1} \bar{\gamma}_{1}\right) z}{\bar{\gamma}_{1} z}-\frac{1}{\gamma_{2}}+\cdots \\
& +\frac{\left(1-\gamma_{\lambda-1} \bar{\gamma}_{\lambda-1}\right) z}{\bar{\gamma}_{\lambda-1} z}-\frac{1}{\gamma_{\lambda}}=d_{\lambda 0}+d_{\lambda 1} z+d_{\lambda 2} z^{2}+\cdots=\phi_{\lambda} .
\end{aligned}
$$

But the first $(\lambda+1)$ terms of this expansion are the same as the first $(\lambda+1)$ terms of the power series expansion (2.1) for $F_{0}(z)$. Consequently,

$$
\begin{aligned}
\left|F_{0}(z)-\phi_{\lambda}\right| & =\left|\sum_{v=0}^{\infty}\left(c_{0 v}-d_{\lambda v}\right) z^{v}\right|=\left|\sum_{v=\lambda+1}^{\infty}\left(c_{0 v}-d_{\lambda v}\right) z^{v}\right| \\
& \leqq \sum_{v=\lambda+1}^{\infty}\left(\left|c_{0 v}\right|+\left|d_{\lambda v}\right|\right) r^{v} \leqq(C+D) \frac{r^{\lambda+1}}{1-r}
\end{aligned}
$$

where

$$
\max \left|c_{0 v}\right|=C, \max \left|d_{\lambda v}\right|=D, \lambda>N, \text { an arbitrarily large number, }
$$

for $z$ in $C_{0}$ (4.1). Since the right-hand expression of (4.4) converges to zero with increasing $\lambda$, the functions $\phi_{\lambda}$ converge uniformly to $F_{0}(z)$ in $C_{0}$.

The following theorem may be proved in an analogous manner.

THEOREM 4.3. A meromorphic function $F_{0}(z)$ can be represented in $C_{0}$ (4.1) by a continued fraction (1.1) provided the coefficients of the power series expansions for the $2 \lambda$ th approximants $(2 \lambda>N)$ of (1.1) are bounded.

Since $F_{0}(z)$ is analytic in $C_{0}(4.1)$, Theorems 4.2 and 4.3 extend the work of Schur beyond analytic functions which have moduli less than one in the unit circle.

It is also noted that if the function $F_{0}(z)$ satisfies the condition $\left|F_{0}(z)\right|<M$ throughout the region $C_{0}$, then $F_{0}(z) / M<1$. Hence $F_{0}(z) / M$ is an analytic function of modulus less than one throughout this region and satisfies the conditions of Schur's continued fraction for analytic functions bounded in the unit circle. The continued fraction which represents $F_{0}(z)$ in $C_{0}$ is therefore

$$
\begin{array}{r}
M \gamma_{0}+\frac{M\left(1-\gamma_{0} \bar{\gamma}_{0}\right) z}{\bar{\gamma}_{0} z}-\frac{1}{\gamma_{1}}+\frac{\left(1-\gamma_{1} \bar{\gamma}_{1}\right) z}{\bar{\gamma}_{1} z}-\frac{1}{\gamma_{2}}+\ldots,\left|\gamma_{p}\right|<1, \\
p=0,1, \ldots .
\end{array}
$$

If one puts $\gamma_{p}=\gamma, p=0,1, \cdots$, in (1.2), the following theorem on a periodic continued fraction is obtained for convergence to the value 
of the power series and its analytic continuation.

TheOREM $4.4 .^{3}$ The periodic continued fraction

$$
\gamma+\frac{(1-\gamma \bar{\gamma}) z}{\bar{\gamma} z}-\frac{1}{\gamma}+\frac{(1-\gamma \bar{\gamma}) z}{\bar{\gamma} z}-\frac{1}{\gamma}+\cdots
$$

converges uniformly to the value of the power series and furnishes the analytic continuation in the cut z-plane. In the cut plane, the function

$$
\frac{1+z-\left(1+z^{2}+(2-4 \gamma \bar{\gamma}) z\right)^{1 / 2}}{2 \bar{\gamma} z}
$$

is regular and is equal to the continued fraction. For $|\gamma|<1$, the cut is along the arc of the unit circle which contains the point $z=1$ between the points $2 \gamma \bar{\gamma}-1 \pm 2 i(\gamma \bar{\gamma}(1-\gamma \bar{\gamma}))^{1 / 2}$. For $|\gamma|>1$, the cut is along the real axis between the points $2 \gamma \bar{\gamma}-1 \mp 2(\gamma \bar{\gamma}(\gamma \bar{\gamma}-1))^{1 / 2}$.

Proof. By a theorem of Perron [5, p. 276], the continued fraction (4.5) converges for all $z$ with the exception of those values which satisfy the condition $\left|\bar{\gamma} z x_{1}-1\right|=\left|\bar{\gamma} z x_{2}-1\right|, x_{1} \neq x_{2}$, where $x_{1}$ and $x_{2}$ are the roots of the equation $\bar{\gamma} z x^{2}-(1+z) x+\gamma=0$. Thus (4.5) diverges when $\left|z-1+\left(1+z^{2}+(2-4 \gamma \bar{\gamma}) z\right)^{1 / 2}\right|=\mid z-1$ $-\left(1+z^{2}+(2-4 \gamma \bar{\gamma}) z\right)^{1 / 2} \mid$, where $1+z^{2}+(2-4 \gamma \bar{\gamma}) z \neq 0$. Then

$$
\begin{aligned}
\left(z-1+\left(1+z^{2}+(2-4 \gamma \bar{\gamma}) z\right)^{1 / 2}\right) e^{i a} & \\
& =\left(z-1-\left(1+z^{2}+(2-4 \gamma \bar{\gamma}) z\right)^{1 / 2}\right) e^{-i a}, a \neq 0, \text { real, }
\end{aligned}
$$

or

$$
(z-1) i \sin a+\left(1+z^{2}+(2-4 \gamma \bar{\gamma}) z\right)^{1 / 2} \cos a=0,
$$

whence

$$
z+\frac{1}{z}=2[\gamma \bar{\gamma}-(1-\gamma \bar{\gamma}) t], \quad-1 \leqq t<1 .
$$

The theorem follows immediately.

5. Convergence of the continued fractions (1.1) and (1.2). Since the development of the continued fractions from an arbitrary power series described in $\$ 2$ and $\$ 3$ is a purely formal process, the question of the convergence of these continued fractions will be further discussed.

${ }^{3}$ This theorem and proof are due to Professor Oskar Perron, who communicated them to the author.

4 Here the approximants $A_{2 p+1} / B_{2 p+1}$ are considered with the common factor $z$ removed. 
The following theorems give convergence regions for large values of $|z|$ and for regions which do not include the origin. They are immediate consequences of the Pringsheim convergence criteria (cf. Perron [5, pp. 254-262; 7]).

THEOREM 5.1. The continued fraction (1.1) converges uniformly over every bounded closed region for which the conditions

$$
\begin{aligned}
\left|c_{2 p-1} \bar{\gamma}_{p-1} z\right| & \geqq\left|k_{p-1}\left(1-\gamma_{p-1} \bar{\gamma}_{p-1}\right) c_{2 p-2} c_{2 p-1} z\right|+1, \\
\left|c_{2 p} k_{p} \gamma_{p}\right| & \geqq\left|c_{2 p-1} c_{2 p}\right|+1, c_{0}=1, p=1,2, \cdots,
\end{aligned}
$$

hold simultaneously. The numbers $c_{p}$ are arbitrary constants different from zero, or functions bounded in the region.

The following convergence regions for the continued fraction (1.1) are obtained by various choices of the numbers $c_{p}$ in the above theorem. The choice $c_{p}=1$ gives Theorem 5.2. If one writes $c_{0}$ $=\left(r_{1}-1\right) / C r_{1}, c_{p}=r_{p} / b_{p}, p=1,2, \cdots$, where the $b_{p}$ are the partial denominators of (1.1), Theorem 5.3 is obtained (cf. [5, p. 262]). The choice $r_{p}=2, p=1,2, \cdots$, yields Theorem 5.4.

Theorem 5.2. If $\left|\gamma_{p}\right| \geqq 2 /\left|k_{p}\right|,\left|\gamma_{p-1}\right|>\left|k_{p-1}\left(1-\gamma_{p-1} \bar{\gamma}_{p-1}\right)\right|$, the continued fraction (1.1) converges uniformly over every bounded closed region for which

$$
|z| \geqq \frac{1}{\left|\gamma_{p-1}\right|-\left|k_{p-1}\left(1-\gamma_{p-1} \bar{\gamma}_{p-1}\right)\right|}, \quad p=1,2, \cdots .
$$

THEOREM 5.3. The continued fraction (1.1) converges uniformly over every bounded closed region for which simultaneously

$$
\begin{aligned}
\left|k_{0}\left(1-\gamma_{0} \bar{\gamma}_{0}\right)\right| \leqq C\left|\gamma_{0}\right|, \quad\left|\frac{1-\gamma_{p} \bar{\gamma}_{p}}{\gamma_{p} \bar{\gamma}_{p}}\right| \leqq \frac{r_{2 p+1}-1}{r_{2 p} r_{2 p+1}}, \\
\left|\frac{1}{k_{p} \bar{\gamma}_{p-1} \gamma_{p} z}\right| \leqq \frac{r_{2 p}-1}{r_{2 p-1} r_{2 p}}, \quad p=1,2, \cdots,
\end{aligned}
$$

where $C$ is an arbitrary constant and the $r_{p}$ are any numbers such that $r_{p}>1$.

THEOREM 5.4. The continued fraction (1.1) converges uniformly over every bounded closed region such that simultaneously $|z|$ $\geqq 2\left(5^{1 / 2}\right) /\left|k_{1} \bar{\gamma}_{0}\right|$ and $|z| \geqq 5 /\left|k_{j}\right|$, where $k_{j}$ denotes the number $k_{p}$ of smallest modulus, and $2 / 5^{1 / 2} \leqq\left|\gamma_{p}\right| \leqq 2 / 3^{1 / 2},\left|\gamma_{p}\right| \neq 1, p=1,2, \cdots$.

By an equivalence transformation the continued fraction (1.1) may be written in the form 


$$
\begin{aligned}
k_{0} \gamma_{0}+k_{0} \gamma_{0}\left(\frac{1}{\left|\gamma_{0}\right|^{2}}-1\right) & {\left[\frac{1}{1}-\frac{1 / k_{1} \bar{\gamma}_{0} \gamma_{1} z}{1}+\frac{1 /\left|\gamma_{1}\right|^{2}-1}{1}\right.} \\
& \left.-\frac{1 / k_{2} \bar{\gamma}_{1} \gamma_{2} z}{1}+\frac{1 /\left|\gamma_{2}\right|^{2}-1}{1}-\ldots\right],
\end{aligned}
$$

provided $\left|\gamma_{p}\right| \neq 0,1, p=0,1, \cdots$. The following convergence region for this expansion is obtained by the parabola theorem [7].

THEOREM 5.5. For values of $\gamma_{p}$ such that $0<\left|\gamma_{p}\right| \leqq 2 / 3^{1 / 2},\left|\gamma_{p}\right| \neq 1$, the continued fraction (5.1) converges uniformly for all $z=r e^{(i \theta)}$ in every bounded closed region such that $r \geqq 2 /\left|k_{p} \gamma_{p} \bar{\gamma}_{p-1}\right|\left[1+R\left[\bar{k}_{p} \bar{\gamma}_{p} \gamma_{p-1}\right.\right.$ $\left.(\cos \theta-\imath \sin \theta)] /\left|k_{p} \gamma_{p} \bar{\gamma}_{p-1}\right|\right]$, provided $\sum\left|d_{p}\right|$ diverges, where $d_{1}=1$, $c_{p}=1 / d_{p} d_{p+1}, c_{2 p-1}=-1 / k_{p} \gamma_{p} \bar{\gamma}_{p-1} z, c_{2 p}=1 /\left|\gamma_{p}\right|^{2}-1, p=1,2, \cdots$.

The conditions of the preceding theorem are, for example, satisfied for $k_{p}=1, \gamma_{p}=10 / 9, p=0,1, \cdots$, in the periodic continued fraction. In this case, (5.1) converges uniformly over every bounded closed region exterior to the cardioid $r=1.62(1+\cos \theta)$. By an application of the theorem of Worpitzky [12] to the expansion (5.1), one obtains the same convergence region as stated in Theorem 5.4. Furthermore, the theorem of Worpitzky states that the moduli of the values of (5.1) and of its approximants do not exceed $\left|k_{0} \gamma_{0}\right|$ $+\left|2 k_{0} / \bar{\gamma}_{0}-k_{0} \gamma_{0}\right|$.

6. The even and odd parts of the expansion (1.1). The even part (cf. $[5$, p. 201]) of $(1.1)$ is

$$
\begin{aligned}
k_{0}\left[\gamma_{0}-\frac{k_{1} \gamma_{1}\left(1-\gamma_{0} \bar{\gamma}_{0}\right) z}{-\bar{\gamma}_{0} k_{1} \gamma_{1} z+1}+\frac{k_{2} \gamma_{2}\left(1-\gamma_{1} \bar{\gamma}_{1}\right) z}{-k_{2} \gamma_{2} z+\gamma_{1}}\right. & \\
& \left.+\frac{k_{3} \gamma_{3}\left(1-\gamma_{2} \gamma_{2}\right) \gamma_{1} z}{-k_{3} \gamma_{3} z+\gamma_{2}}+\cdots\right]
\end{aligned}
$$

or

$$
\begin{aligned}
k_{0} / w\left[\gamma_{0} w-\frac{k_{1} \gamma_{1}\left(1-\gamma_{0} \bar{\gamma}_{0}\right)}{-\gamma_{0} k_{1} \gamma_{1} / w+w}+\right. & \frac{k_{2} \gamma_{2}\left(1-\gamma_{1} \bar{\gamma}_{1}\right)}{-k_{2} \gamma_{2} / w+\gamma_{1} w} \\
& \left.+\frac{k_{3} \gamma_{3}\left(1-\gamma_{2} \bar{\gamma}_{2}\right) \gamma_{1}}{-k_{3} \gamma_{3} / w+\gamma_{2} w}+\cdots\right],
\end{aligned}
$$

where $w=1 / z^{1 / 2}$.

Expansion -(6.1) is of the same form first considered by Euler [1], who derived it from a given power series, but the convergence of this 
expansion does not seem to have been studied. The convergence theorems of $\S 4$ and $\S 5$ are applicable to (6.1) since the approximants of (6.1) are the even approximants of (1.1). In addition, conditions which insure the convergence of (6.1) in the neighborhood of the origin will now be found.

Theorem 6.1. If $0<m \leqq\left|k_{p}\right| \leqq 1,0<m \leqq\left|\gamma_{p}\right|<1, p=0,1, \cdots$, the continued fraction (6.1) converges uniformly over every bounded closed region within

$$
|z| \leqq \frac{m^{2}}{\left(1+(1+m)^{1 / 2}\right)^{2}} .
$$

It also converges for all $z$ in the region

$$
|z| \geqq \frac{1}{\left(\left(1+m^{3}\right)^{1 / 2}-1\right)^{2}},
$$

and uniformly in every bounded closed region interior to this region.

Proof. By the hypotheses on the $k_{p}$ and $\gamma_{p}$, for $|w|=r$, $\left|k_{2} \gamma_{2}\left(1-\gamma_{1} \bar{\gamma}_{1}\right)\right| \leqq 1, \quad\left|\gamma_{p-2}\left(1-\gamma_{p-1} \bar{\gamma}_{p-1}\right) k_{p} \gamma_{p}\right| \leqq 1, p=3,4, \cdots$, $\left|-k_{p} \gamma_{p} / w+\gamma_{p-1} w\right| \geqq-\left|k_{p} \gamma_{p} / w\right|+\left|\gamma_{p-1} w\right| \geqq-1 / r+m r \geqq 2, \quad p=2$, $3, \cdots$, provided $r \geqq 1\left(1+(1+m)^{1 / 2}\right) / m$ or $\left.|z| \leqq m^{2} /(1+m)^{1 / 2}\right)^{2}$. Also, for the first partial denominator of (6.1), $\left|-\gamma_{0} k_{1} \gamma_{1} / w+w\right|$ $\geqq r-1 / r \geqq 2$ provided $r \geqq 1+2^{1 / 2}$ or $|z| \leqq 1 /\left(1+2^{1 / 2}\right)^{2}$. The region (6.3) is contained in this region. Consequently by the Pringsheim convergence criteria, (6.1) converges uniformly over every bounded closed region within (6.3).

Similarly, one obtains the convergence region (6.4), since $\mid-k_{p} \gamma_{p} / w$ $+\gamma_{p-1} w|\geqq-| \gamma_{p-1} w|+| k_{p} \gamma_{p} / w \mid \geqq-r+m^{2} / r \geqq 2, p=2,3, \cdots$, provided $r \leqq-1+\left(1+m^{2}\right)^{1 / 2}$ or $|z| \geqq 1 /\left(\left(1+m^{2}\right)^{1 / 2}-1\right)^{2}$. For the first partial denominator of (6.1), $\left|-\gamma_{0} k_{1} \gamma_{1} / w+w\right| \geqq-r+m^{3} / r$ $\geqq 2$, provided $|z| \geqq 1 /\left(\left(1+m^{3}\right)^{1 / 2}-1\right)^{2}$. Here $1 /\left(\left(1+m^{3}\right)^{1 / 2}-1\right)^{2}$ $\geqq 1 /\left(\left(1+m^{2}\right)^{1 / 2}-1\right)^{2}$.

Since in the expansion of a power series (2.1) into a continued fraction (1.1), it is always possible to choose the numbers $k_{p}$ such that $1<\left|\gamma_{p}\right| \leqq N, p=0,1, \cdots$, where $N$ is an arbitrary positive constant, the following theorem insures the uniform convergence of the even part of such a continued fraction in the interior of a circular region about the origin.

THeOREM 6.2. If $0<\left|k_{p} \gamma_{p}\right| \leqq M,\left|1-\gamma_{p} \bar{\gamma}_{p}\right| \leqq K, 1<\left|\gamma_{p}\right| \leqq N$, $p=0,1, \cdots$, where $M, K$, and $N$ are arbitrary positive constants such that $K \geqq(N-1) / 4,(6.1)$ converges uniformly over every bounded closed 
region within

$$
|z| \leqq \frac{1}{2 M N(1+2 K)} .
$$

PROOF. For $|w|=r,\left|k_{p+1} \gamma_{p+1}\left(1-\gamma_{p} \bar{\gamma}_{p}\right) \gamma_{p-1}\right| \leqq M K N \leqq\left(r^{2}-2 M N\right) / 4$ $\leqq\left[\left|k_{p+1} \gamma_{p+1} k_{p} \gamma_{p} / w^{2}\right|+\left|\gamma_{p} \gamma_{p-1} w^{2}\right|-\left|k_{p+1} \gamma_{p+1} \gamma_{p-1}\right|-\left|k_{p} \gamma_{p}{ }^{2}\right|\right] / 4$ $\leqq\left|\left(-k_{p+1} \gamma_{p+1} / w+\gamma_{p} w\right)\left(-k_{p} \gamma_{p} / w+\gamma_{p-1} w\right)\right| / 4, \quad p=2, \quad 3, \cdots$. Hence by the Pringsheim convergence criteria, (6.1) converges uniformly over the region $|w|^{2}=r^{2} \geqq 2 M N+4 M K N$, or $|z| \leqq 1 / 2 M N(1+2 K)$, provided $K \geqq(N-1) / 4$. The latter condition is necessitated by a slight irregularity at the beginning of the continued fraction. Here, for the Pringsheim criteria to hold, $\left|k_{2} \gamma_{2}\left(1-\gamma_{1} \bar{\gamma}_{1}\right)\right| \leqq M K \leqq\left(r^{2}-M-M N^{2}\right) / 4 \leqq\left[\left|\gamma_{0} k_{1} \gamma_{1} k_{2} \gamma_{2} / w^{2}\right|+\left|\gamma_{1} w^{2}\right|\right.$ $\left.-\left|k_{2} \gamma_{2}\right|-\left|k_{1} \gamma_{0} \gamma_{1}^{2}\right|\right] / 4 \leqq\left|\left(-\gamma_{0} k_{1} \gamma_{1} / w+w\right)\left(-k_{2} \gamma_{2} / w+\gamma_{1} w\right)\right| / 4, \quad$ so that $r^{2} \geqq 4 M K+M\left(1+N^{2}\right)$, or $|z| \leqq 1 /\left[4 M K+M\left(1+N^{2}\right)\right]$. The circular region (6.5) is contained in or coincides with this region if $K \geqq(N-1) / 4$.

As an illustration of this theorem, consider the expansion of the function (3.5) with $\gamma_{p}=2, p=0,1, \cdots$. By the algorithm ( $\$ 3$ ), $k_{0}=1 / 2, \quad k_{p}=(-1)^{p+1} / 3^{p}, p=1,2, \cdots$ Then, for $M=1, K=3$, $N=2$, the continued fraction (6.1) converges for $|z| \leqq 1 / 28$.

If $0<\left|\gamma_{p}\right| \leqq N, 1 \leqq\left|k_{p} \gamma_{p}\right| \leqq M$, the following theorem gives convergence for large values of $|z|$.

Theorem 6.3. If $1 \leqq\left|k_{p} \gamma_{p}\right| \leqq M,\left|1-\gamma_{p} \bar{\gamma}_{p}\right| \leqq K, 0<\left|\gamma_{p}\right| \leqq N$, $p=0,1, \cdots$, where $M, K$, and $N$ are arbitrary positive constants such that $N \geqq 1 / \gamma_{0},(6.1)$ converges for all $z$ in the region

$$
|z| \geqq 2 M N(1+2 K)
$$

and uniformly in every bounded closed region interior to this region.

PROOF. For $|w|=r,\left|k_{p+1} \gamma_{p+1}\left(1-\gamma_{p} \bar{\gamma}_{p}\right) \gamma_{p-1}\right| \leqq M K N \leqq\left(1 / r^{2}-2 M N\right) / 4$ $\leqq\left[\left|k_{p+1} \gamma_{p+1} k_{p} \gamma_{p} / w^{2}\right|+\left|\gamma_{p} \gamma_{p-1} w^{2}\right|-\left|k_{p+1} \gamma_{p+1} \gamma_{p-1}\right|-\left|k_{p} \gamma_{p}^{2}\right|\right] / 4$ $\leqq\left|\left(-k_{p+1} \gamma_{p+1} / w+\gamma_{p} w\right)\left(-k_{p} \gamma_{p} / w+\gamma_{p-1} w\right)\right| / 4$. Hence (6.1) converges for $r^{2} \leqq 1 / 2 M N(1+2 K)$ or $|z| \geqq 2 M N(1+2 K)$. By a method analogous to that used in Theorem 6.2, for the Pringsheim criteria to hold for the irregularity at the beginning of (6.1), it can be shown that $|z| \geqq\left[4 M K+M\left(1+N \gamma_{0}\right)\right] / \gamma_{0}$. This region lies interior to or coincides with (6.6) provided $N \geqq 1 / \gamma_{0}$.

The even part of (1.1) is similar in form to the expansion

$$
1+d_{0} z+\frac{z}{1+d_{1} z}+\frac{z}{1+d_{2} z}+\cdots
$$


discussed by Thron [8]. For

$$
\begin{aligned}
& k_{0} \gamma_{0}=1, \quad d_{0}=\text { an arbitrary finite number, } \\
& -k_{0} k_{1} \gamma_{1}\left(1-\gamma_{0} \bar{\gamma}_{0}\right)=1, \quad d_{1}=-\bar{\gamma}_{0} k_{1} \gamma_{1}, \\
& \frac{k_{n} \gamma_{n}\left(1-\gamma_{n-1} \bar{\gamma}_{n-1}\right)}{\gamma_{n-1}}=1, \quad d_{n}=\frac{-k_{n} \gamma_{n}}{\gamma_{n-1}}, \quad n=2,3, \cdots,
\end{aligned}
$$

the expansions (6.1) and (6.7) are identical in form. For an arbitrary power series $1+\left(1+d_{0}\right) z+c_{2} z^{2}+c_{3} z^{3}+\cdots$ the $k_{p}$ and $\gamma_{p}$, $p=0,1, \cdots$, may be found by (3.2) and (6.8) such that the even part of the resulting continued fraction (1.1) will be of exactly the same form as (6.7). However, since the law of formation of the coefficients $d_{p}$ for the expansion (6.7) (cf. [8]) is different from that used in the algorithm of $\$ 3$ and in the formulas (6.8), the coefficients $d_{p}$ in the continued fractions (6.7) corresponding to the same power series, but found by the two different methods, are in general different.

The odd part (cf. $[5$, p. 201]) of (1.1) is

$$
\begin{gathered}
\frac{k_{0} \bar{\gamma}_{0} z}{\bar{\gamma}_{0}^{2} z}+\frac{k_{0}\left(1-\gamma_{0} \bar{\gamma}_{0}\right) \bar{\gamma}_{1}}{-\bar{\gamma}_{1}+k_{1} \bar{\gamma}_{0} z}+\frac{k_{1}\left(1-\gamma_{1} \bar{\gamma}_{1}\right) \bar{\gamma}_{0} \bar{\gamma}_{2} z}{-\bar{\gamma}_{2}+k_{2} \bar{\gamma}_{1} z} \\
+\frac{k_{2}\left(1-\gamma_{2} \bar{\gamma}_{2}\right) \bar{\gamma}_{1} \bar{\gamma}_{3} z}{-\bar{\gamma}_{3}+k_{3} \bar{\gamma}_{2} z}+\ldots
\end{gathered}
$$

By methods similar to those for the even part (6.1) of (1.1), one may find convergence regions for the odd part (6.9). Such regions depend on the conditions which the numbers $k_{p}$ and $\gamma_{p}$ satisfy, as, for instance, in the following theorem.

Theorem 6.4. If $0<\left|k_{p}\right| \leqq M, \quad\left|1-\gamma_{p} \bar{\gamma}_{p}\right| \leqq K, \quad 1<\left|\gamma_{p}\right| \leqq N$, $p=0,1, \cdots$, where $M, K$, and $N$ are positive constants, such that $1 \geqq 16 K M^{2} N^{4}>1-(1-N /(1+2 K))^{2}$, (6.9) converges uniformly over every bounded closed region in the annulus

$$
\frac{8 K M N}{1-\left(1-16 K M^{2} N^{4}\right)^{1 / 2}} \leqq|z| \leqq \frac{1}{2 M N^{2}(1+2 K)} .
$$

7. Transformations of the continued fraction (1.1). For the meromorphic function

$$
F(z)=\frac{\delta_{0}+z F_{0}(z)}{\delta_{0}-z F_{0}(z)}
$$

where $F(z)$ has no poles in $|z|<1, \delta_{0}$ is a constant different from zero, 
and $F_{0}(z)$ is a meromorphic function, with the use of the functions

$$
h_{p}(z)=\frac{\delta_{k}-f_{k}}{\delta_{k}+z f_{k}}, \quad k=0,1, \cdots, p,
$$

as in [2], the following continued fraction is obtained (where the right-half plane has been mapped on the unit circle).

THEOREM 7.1. The infinite continued fraction

$$
\begin{gathered}
\frac{1+z}{1-z}+\frac{2 z\left(\delta_{0}^{\prime}-\gamma_{0}^{\prime}\right)}{\delta_{0}^{\prime}-\delta_{1} z}+\frac{\left(\delta_{1}+\gamma_{0}^{\prime}\right)\left(\delta_{1}^{\prime}-\gamma_{1}^{\prime}\right) z}{\delta_{1}^{\prime}-\delta_{2} z} \\
+\frac{\left(\delta_{2}+\gamma_{1}^{\prime}\right)\left(\delta_{2}^{\prime}-\gamma_{2}^{\prime}\right) z}{\delta_{2}^{\prime}-\delta_{3} z}+\cdots
\end{gathered}
$$

where

$$
\delta_{p+1}=\frac{\delta_{p}^{\prime}-\gamma_{p}^{\prime}}{1-\delta_{p}^{\prime} \bar{\gamma}_{p}^{\prime}}, \quad \delta_{p}^{\prime}=\frac{\delta_{p}}{k_{p}}, \quad \gamma_{p}^{\prime}=\frac{\gamma_{p}}{k_{p}}, \quad p=0,1, \cdots,
$$

and where the $k_{p}$ are chosen so that $\left|\gamma_{p}\right| \neq 1, \delta_{p}^{\prime} \neq \gamma_{p}^{\prime}$, converges uniformly over every bounded closed domain in $C_{0}(4.1)$ to the meromorphic function $F(z)$ (7.1), provided the coefficients of the power series expansion for the $2 \lambda$ th approximants of (7.2) remain bounded, for $\lambda$ sufficiently large. The expansion (7.2) is finite when the $\gamma_{p}$ form a finite sequence.

By the transformation

$$
w=\frac{4 r z}{(r-z)^{2}}, \quad z=r \frac{(1+w)^{1 / 2}-1}{(1+w)^{1 / 2}+1},
$$

which maps the interior of $|z|=r$ on the $w$-plane exterior to the cut from -1 to $-\infty$, where $r$ is defined by (4.1), one obtains the convergence to the function $F$ of the continued fraction

$$
\begin{aligned}
\frac{(1-r)+(1+r)(1+w)^{1 / 2}}{(1+r)+(1-r)(1+w)^{1 / 2}} & +\frac{2\left(\delta_{0}^{\prime}-\gamma_{0}^{\prime}\right) r w}{\left(\delta_{0}^{\prime}+\delta_{1} r\right)+\left(\delta_{0}^{\prime}-\delta_{1} r\right)(1+w)^{1 / 2}} \\
& +\frac{\left(\delta_{1}+\gamma_{0}^{\prime}\right)\left(\delta_{1}^{\prime}-\gamma_{1}^{\prime}\right) r w}{\left(\delta_{1}^{\prime}+\delta_{2} r\right)+\left(\delta_{1}^{\prime}-\delta_{2} r\right)(1+w)^{1 / 2}}+\ldots
\end{aligned}
$$

provided the coefficients of the power series expansions for the $2 \lambda$ th approximants of this continued fraction remain bounded for $\lambda$ sufficiently large. The continued fraction converges uniformly over every 
bounded closed region which lies in the w-plane exterior to the cut along the real axis from -1 to $-\infty$.

In (7.4), let $\delta_{0}=1,\left|\delta_{p}\right|=1, k_{p-1}=1, p=1,2, \cdots$. One thus obtains an extension of the theorem in [9]: If $c$ is a nonzero constant, $-\infty<g_{p}<\infty, g_{p} \neq 1, a_{p-1}=1+i s_{p-1}, b_{p-1}=1-i s_{p-1},-\infty<s_{p-1}<\infty$, $p=1,2, \cdots$, the continued fraction

$$
\begin{aligned}
\frac{c\left[(1-r)+(1+r)(1+w)^{1 / 2}\right]}{\left(a_{0}+b_{0} r\right)+\left(a_{0}-b_{0} r\right)(1+w)^{1 / 2}} & \\
& +\frac{4 g_{1} r w}{\left(a_{1}+b_{1} r\right)+\left(a_{1}-b_{1} r\right)(1+w)^{1 / 2}} \\
& +\frac{4\left(1-g_{1}\right) g_{2} r w}{\left(a_{2}+b_{2} r\right)+\left(a_{2}-b_{2} r\right)(1+w)^{1 / 2}} \\
& +\frac{4\left(1-g_{2}\right) g_{3} r w}{\left(a_{3}+b_{3} r\right)+\left(a_{3}-b_{3} r\right)(1+w)^{1 / 2}}+\cdots
\end{aligned}
$$

converges uniformly over every bounded closed domain in the w-plane exterior to the cut along the real axis from -1 to $-\infty$ to the meromorphic function $F(z)$, provided the coefficients of the series expansions of the $2 \lambda$ th approximants of the continued fraction are bounded for $\lambda$ sufficiently large.

\section{BIBLIOGRAPHY}

1. L. Euler, De fractionibus continuis, Introductio in analysin infinitorum, chap. 18, vol. 1, 1748 (Opera Omnia, Series Prima, vol. 8, pp. 362-390).

2. E. Frank, On the real parts of the zeros of complex polynomials and applications to continued fraction expansions of analytic functions, Trans. Amer. Math. Soc. vol. 62 (1947) pp. 272-283.

3. G. Mittag-Leffler, Ofversigt af Kongl-Vetenskaps-akademiens Forhandlingar, vol. 34 (1877).

4. - Sur la representation analytique des fonctions uniformes monògenes d'une variable indépendante, Acta Math. vol. 4 (1884) pp. 1-79.

5. O. Perron, Die Lehre von den Kettenbritchen, Leipzig, Teubner, 1929.

6. I. Schur, Über Potenzreihen, die im Innern des Einheitskreises beschränkt sind, J. Reine Angew. Math. vol. 147 (1917) pp. 205-232; vol. 148 (1918) pp. 122-145.

7. W. T. Scott and H. S. Wall, $A$ convergence theorem for continued fractions, Trans. Amer. Math. Soc. vol. 47 (1940) pp. 155-172.

8. W. J. Thron, Some properties of continued fractions $1+d_{0} z+K\left(z / 1+d_{n} z\right)$, Bull. Amer. Math. Soc. vol. 54 (1948) pp. 206-218.

9. H. S. Wall, Continued fraction expansions for functions with positive real parts, Bull. Amer. Math. Soc. vol. 52 (1946) pp. 138-143.

10. K. Weierstrass, Zur Theorie der eindeutigen analytischen Functionen, Abhandlungen der Königl. Akademie der Wissenschaften (1876) p. 11 (Werke pp. 77124). 
11. - Über einen functionentheoretischen Satz des Herrn. G. Mittag-Leffler, Monatsbericht der Königl. Akademie der Wissenschaften (1880) (Werke, pp. 189199).

12. J. Worpitzky, Untersuchungen ibber die Entwickelung der Monodronen und mongenen Functionen durch Kettenbriuche, Friedrichs Gymnasium und Realschule, Jahresbericht (Berlin) 1865.

UNIVERSITY OF ILLINOIS

\section{ARBITRARY MAPPINGS}

H. D. BLOCK AND BUCHANAN CARGAL

The results of this paper generalize some results of $\mathrm{H}$. Blumberg. ${ }^{1}$

Let $X$ be a set of elements and let $\mathfrak{N}$ be a collection of nonempty subsets of $X$. We assume that there is, in $\mathfrak{R}$, a countable subcollection: $N^{1}, N^{2}, \cdots, N^{n}, \cdots$ with the property that, for each $N$ of $\mathfrak{R}$ and each $x$ of $N$, there is an integer $k$, such that $x \in N^{k}$ and $N^{k} \subseteq N$. In the remainder of this paper the letter $N$ with a superscript will always denote a member of the set $N^{1}, N^{2}, \cdots, N^{n}, \cdots$. The letter $N$ with or without subscripts will always denote a member of $\mathfrak{N}$, and the letter $x$ will denote an element of $X$. The symbol $N(x)$ denotes a set $N$ which contains $x$.

Let the statements of the preceding paragraph be repeated, replacing $X$ by $Y, \mathfrak{R}$ by $\mathfrak{M}, N$ by $M, x$ by $y$.

Let a correspondence, $f$, be given which to each $x$ assigns a nonempty subset (denoted by $f(x)$ ) of $Y$. If $V \subseteq Y$, then $f^{-1}(V)$ denotes the set of all $x$ such that $f(x) \cdot V \neq 0$.

Definitions. Let $S$ be a subset of $X$. Then $S$ is nowhere dense if, for each $N$, there is an $N_{1} \subseteq N$ with $N_{1} \cdot S=0$. A set is exhaustible if it is the union of a countable collection of nowhere dense sets. A set is residual if it is the complement (with respect to $X$ ) of an exhaustible set. A set is inexhaustible if it is not exhaustible. The point $x$ is said to be a point of exhaustible f-approach if there is a $y$ in $f(x)$, an $M(y)$, and an $N(x)$ such that $\left[f^{-1}(M(y))\right] \cdot N(x)$ is exhaustible.

Presented to the Society, April 26, 1952; received by the editors March 7, 1952.

1 New properties of all real functions, Trans. Amer. Math. Soc. vol. 24 (1922) pp. 113-128. Also Arbitrary point transformations, Duke Math. J. vol. 11 (1944) pp. 671685. The definition of $f$-approach and $\lambda$ approach that we use here are essentially those introduced by Blumberg. 